Neurosurg Focus 14 (1):Article 5, 2003, Click here to return to Table of Contents

\title{
Correction of adult scoliosis via a posterior-only approach
}

\author{
Caleb R. Lippman, M.D., Caple A. Spence, M.D., A. Samy Youssef, M.D., Ph.D., \\ AND DAVID W. CAHILl, M.D. \\ Department of Neurosurgery, University of South Florida, Tampa, Florida
}

\begin{abstract}
Object. Adult scoliosis is a pathologically different entity from adolescent idiopathic scoliosis. The curves are more rigid, and rotational deformity and multilevel sagittal vertebral slippages compound the coronal malalignment. To correct these deformities, a surgical anterior release procedure is usually required, as well as posterior instrumentationassisted fusion. This exposes the patient to the risks of a second procedure and of a thoracotomy or laparotomy. To decrease these risks, the authors have performed an anterior release, posterior release, and reduction via a posterioronly approach. The purpose of this study was to analyze quantitatively the degree of pre- and postoperative coronal deformity, the extent of correction, and related complications.

Methods. Data obtained in 20 patients with adult scoliosis were retrospectively studied. Patients presented with persistent back or lower-extremity pain, progressive deformity, or progressive neurological deficit. Sixteen patients underwent Gill-type laminectomy, radical discectomy (including fracture of any anterior and lateral osteophytes), and posterior lumbar interbody fusion (PLIF) of all apical and adjacent segments. One to four anterior release procedures were performed in each patient. Posterior instrumentation was placed over three to 15 levels. Autograft was obtained from the laminectomy sites and posterior iliac crest for fusion. There were no deaths; all patients were followed for a minimum of 1 year. The mean coronal Cobb angle improved from $36^{\circ}$ to $14.7^{\circ}$. All spondylolisthetic lesions were reduced to at least Grade I. At the most recent follow-up examination, evidence of fusion was demonstrated in all patients. Reoperation for adjacent-segment failure, cephalad to the highest level of fusion, was required in two cases.

Conclusions. In many cases of adult scoliosis, a satisfactory multiplanar correction may be obtained via a single posterior approach and by using extended PLIF techniques. Cephalad adjacent-segment failure remains a significant problem in patients with osteoporosis, and routine extension of posterior instrumentation to the upper thoracic spine should be considered in these cases.
\end{abstract}

\section{KEY WORDS - adult degenerative scoliosis - adult idiopathic scoliosis • posterior approach • spinal deformity - posterior lumbar interbody fusion}

Adult patients with scoliosis often present with a rigid curve, which can be associated with complex rotational deformity and multilevel sagittal spondiolistheses. ${ }^{12,18,19,21 \text {, }}$ 23,24,29,33,34 The disease often progresses over time and can lead to cosmetic and psychological problems, back pain, radiculopathy, spinal claudication, and ultimately spinal

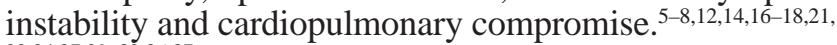
22,24,27,29-32,36,37 Although primary treatment is conservative, including spinal orthoses, physical rehabilitation, and pain control, these measures often fail, or patients may not be candidates for such therapy. $5,6,15,18,19,21,22,29-32,36$ In such cases, surgery is recommended. ${ }^{5-7,15,18-21,25,27,29-32,36}$ Surgical interventions have included posterior instrumentation and fusion alone, $5,6,8,13,17,21,27,29,30,32,36$ anterior instrumentation and fusion alone, $, 5,6,13,17,19,20,27,29$ and anterior release (to restore spinal flexibility) combined with posterior fusion. ${ }^{5-7}$, $13,16,17,19,29,30,36$

Although all methods have been successful, surgical correction is hampered by the rigidity of the curvatures,

Abbreviation used in this paper: PLIF = posterior lumbar interbody fusion.

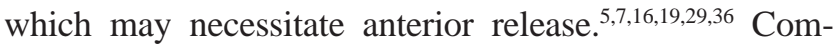
bined approaches, while often more successful in treating the deformity, have the increased risks in elderly and frail patients of a second significant operation, including a second induction of general anesthesia, increased operating time, and excessive blood loss. ${ }^{6,29}$ Surgery in adults with scoliosis is technically more demanding than similar surgery in adolescents. ${ }^{27,32,36} \mathrm{In}$ adults the curves are more rigid, the bones more osteopenic (making instrumentation more difficult), and the patients generally more frail and in poorer health than adolescents. ${ }^{1,16,19-21,25,27,30,32,36}$ Higher complication rates in adults have been documented. ${ }^{5,13,27}$, 29,32,36 Complications inherent to the retroperitoneal approach include abdominal visceral, great vessel, and superior hypogastric nerve plexus injury. ${ }^{11,38}$ Transthoracic approach-induced complications include injury to the great vessels, ${ }^{35,38}$ pulmonary complications, ${ }^{35,38}$ chylothorax, ${ }^{4}$ and the postthoracotomy pain syndrome. ${ }^{26}$

In an effort to decrease the risks of combined procedures while maintaining the ability to satisfactorily reduce complex deformities, we performed anterior and posterior release, instrumentation placement, and reduction of spinal deformity via a single posterior approach. 


\section{CLINICAL MATERIAL AND METHODS}

We retrospectively studied the medical records of 20 patients with scoliosis, as well as radiographs obtained in 17 , all 20 of whom underwent anterior and posterior release procedures via a posterior-only approach. This study does not include patients with primary spondylolisthesis, other sagittal deformities, tumors, or infections.

\section{Patient Population}

We reviewed the cases of 20 patients with adult scoliosis. There were 17 women and 3 men, ranging in age from 34 to 84 years (mean 61 years), whose most recent physical exam and radiographs were between 12 and 17 months (mean 13 months) postsurgery.

\section{Signs and Symptoms}

All patients presented with severe, progressive back pain. Other complaints included radiculopathy (nine cases), progression of scoliosis (six cases), imbalance while walking (five cases), and a progressive neurological deficit (two cases). On examination, 11 patients were neurologically intact, four suffered significant weakness, one had changes in sensorium, and seven exhibited abnormal or pathological reflexes. Primary degenerative lumbar or thoracic scoliosis was presented in nine patients, postsurgical scoliotic degeneration in seven, untreated adolescent scoliosis with secondary adult degeneration in three, and neurogenic scoliosis in one patient. Associated sagittalplane malalignment (olistheses) was revealed in three cases, and associated antero- and retrolisthesis in two cases.

\section{Neuromaging Studies}

Preoperatively obtained whole-spine, plain, standing coronal and sagittal radiographs with a 36-in cassette were assessed for evidence of kyphotic or lordotic deformity, coronal alignment, sagittal malalignment, and plum line assessment. Lateral bending and flexion-extension radiographs allowed for evaluation of spinal flexibility and differentiation of primary and secondary curves. Spinal magnetic resonance imaging and/or computerized tomography scanning (with bone and soft-tissue window densities) were performed to rule out other cases of spinal deformity such as tumor and infection. In the immediate postoperative period, all patients underwent plain radiography of the spine to determine alignment and the accuracy of instrumentation placement. All patients underwent plain radiography at 6 weeks, 12 weeks, 6 months, 12 months, and then annually to assess spinal alignment and the extent of osseous fusion. Sagittal and coronal alignment was determined using the Cobb method. ${ }^{3}$

\section{Indication for Surgery}

Patients who presented with intractable pain, progressive neurological deficit, or significant deformity were offered corrective surgery. Otherwise, they were treated conservatively with orthoses and pain control medication, and they underwent plain radiographic follow-up evaluation every 6 months. Patients with progressive deformity or in whom conservative therapy failed were then offered corrective surgery.

\section{Surgical Procedures}

Patients were positioned prone on the operating table and their abdomen allowed to hang freely. The spine was exposed subperiosteally via a midline posterior route such that the posterior elements including the transverse processes were clearly visible bilaterally. Intraoperative fluoroscopy was used to confirm the spinal level, as well as to aid in instrumentation placement and evaluation of spinal alignment.

Four patients did not undergo PLIFs: one patient with progression of adolescent scoliosis, in whom the spine was flexible and anterior release was not required; one with neurogenic scoliosis secondary to spina bifida and a tethered cord, in whom PLIF was deemed too risky to the low-lying conus medullaris; and two in whom, after removal of the old hardware, previous surgical fusions had failed, in whom it was found that their spines were flexible enough to manipulate without PLIF. In these four patients, the deformities were corrected by performing posterior surgery and instrumentation-assisted fusion.

Sixteen patients required anterior release to manipulate the spines. This facet was planned to be performed at the apical levels of the scoliotic curves, as well as at any adjacent levels with significant disc degeneration or vertebral body collapse. The following sequence was followed: Gill laminectomies (that is, the removal of all posterior elements except the superior articulating facet and the pedicles), radical discectomies, and anterior and lateral osteophyte fracture. The anterior column was then reconstructed by placing bilateral titanium or carbon fiber cages, which were filled with cancellous posterior iliac crest autograft obtained through a separate facial incision. If a cage could not be safely placed, either because one of the vertebral endplates had fractured or the disc space was too collapsed to accommodate a cage, then autologous bone chips were packed within the disc space. The PLIF was performed at one to four levels in each patient. Bilateral cage-assisted PLIF was performed 24 times (48 cages placed), unilateral cage-assisted PLIF 10 times, bilateral bone chip-augmented PLIF four times, and unilateral bone chip-augmented PLIF two times. The L3-4 level was the most common (13 times) interspace treated by PLIF, followed by L2-3 and L4-5 (10 times each), L1-2 (four times), L5-S1 (two times), and T12-L1 (once).

After the PLIF was complete, posterior instrumentation was applied. Multiple points of fixation were achieved posteriorly by inserting pedicle screws primarily; hooks were used when pedicle fixation was not possible because the pedicles were too small, or as supplemental fixation. In one patient with very advanced osteoporosis, methylmethacrylate cement-assisted vertebroplasty was performed to ensure adequate screw pullout resistance. Posterolateral intertransverse fusion was performed over the instrumented levels; the material consisted of the remainder of the cancellous bone obtained from the hip and the morselized posterior elements obtained from the Gill laminectomies. Posterior fusion was performed over three to 15 levels. The levels for instrumentation were determined by the curve characteristics with the uppermost point of fixation being two to three levels above the apical segment. In six patients an electrical bone graft stimulator was placed after final hardware insertion to encourage arthrodesis. 


\section{Posterior correction of adult scoliosis}

\section{Postoperative Management and Follow Up}

Only the severely osteoporotic patient in whom intraoperative vertebroplasty was required wore an external orthosis. All patients received bedside physical therapy and inpatient or outpatient rehabilitation as needed. Physical examinations were focused on neurological status and operative sites. Radiographic measurements were performed for the regional Cobb angles in the coronal and sagittal plane. Patients were seen in the clinic at 6 weeks, 12 weeks, 6 months, 12 months, and annually thereafter. Examination included queries about pain, ambulation, daily activities, and work.

\section{RESULTS}

No patient died during the immediate postoperative or follow-up period. Patients in the series had the most recent follow-up exam and radiographs were between 12 and 17 months (mean 13 months) postsurgery.

\section{Spinal Deformities}

All preoperative and most recent follow-up lateral radiographs were evaluated. (Three cases, for which file folders could not be found, were excluded from these calculations.) In 17 patients, 21 curves were surgically corrected. Eighteen of the 21 corrected curves were in the lumbar spine (Figs. 1 and 2); the remainder were in the thoracic spine (Fig. 1). The mean preoperative coronal Cobb angle for all 21 curves, was $36^{\circ}$ (range $12-73^{\circ}$ ), and the mean postoperative Cobb angle was $14.7^{\circ}$ (range $4-47^{\circ}$ ). The mean correction was $21.3^{\circ}$ (range $5-39^{\circ}$ ). The mean preoperative Cobb angles were $35.1^{\circ}$ (range $10-73^{\circ}$ [18 cases]) and $53.3^{\circ}$ (range $45-65^{\circ}$ [three cases]) in the lum- bar and thoracic spines, respectively, and were corrected to $13.8^{\circ}$ (range $6-47^{\circ}$ ) in the lumbar spine and $24.3^{\circ}$ (range $10-43^{\circ}$ ) in the thoracic spine. Three patients presented with lateral sagittal vertebral displacements involving five levels. There were two Grade I and three Grade II spondylolistheses; all Grade I were reduced completely (Grade 0), and all Grade II were reduced to Grade I. Plain radiography demonstrated evidence of osseous fusion in all patients. Two patients presented with three anterolisthesis and three retrolisthesis combined, all Grade II. These were repaired to Grade I after arthrodesis.

\section{Procedure-Related Complications}

There were no deaths. Three patients suffered nerve root injuries, as evidenced by radiculopathy or weakness in the nerve's distribution. Postoperative computerized tomography scanning of the affected levels was performed to rule out any nerve impingement by instrumentation, and no patient required reoperation for this reason. The nerve root injuries were treated conservatively, and all resolved. There were no cases of spinal cord injuries.

Two patients were returned to the operating room when failure of the adjacent cephalad segment was discovered. At 3-month follow-up examination, plain radiography in one patient revealed thoracic kyphosis and fractured L-2 pedicles, the uppermost level of her construct. She underwent reoperation to extend the instrumentation and arthrodesis up to T-9, and she has fared well since. The other patient presented to an outside hospital with acutely progressive lower-extremity paraparesis. The workup revealed a T-8 compression fracture above the highest level (T-9) of her prior instrumentation. She underwent extension of the fusion to T-1; her neurological status returned to baseline, and she has done well since.

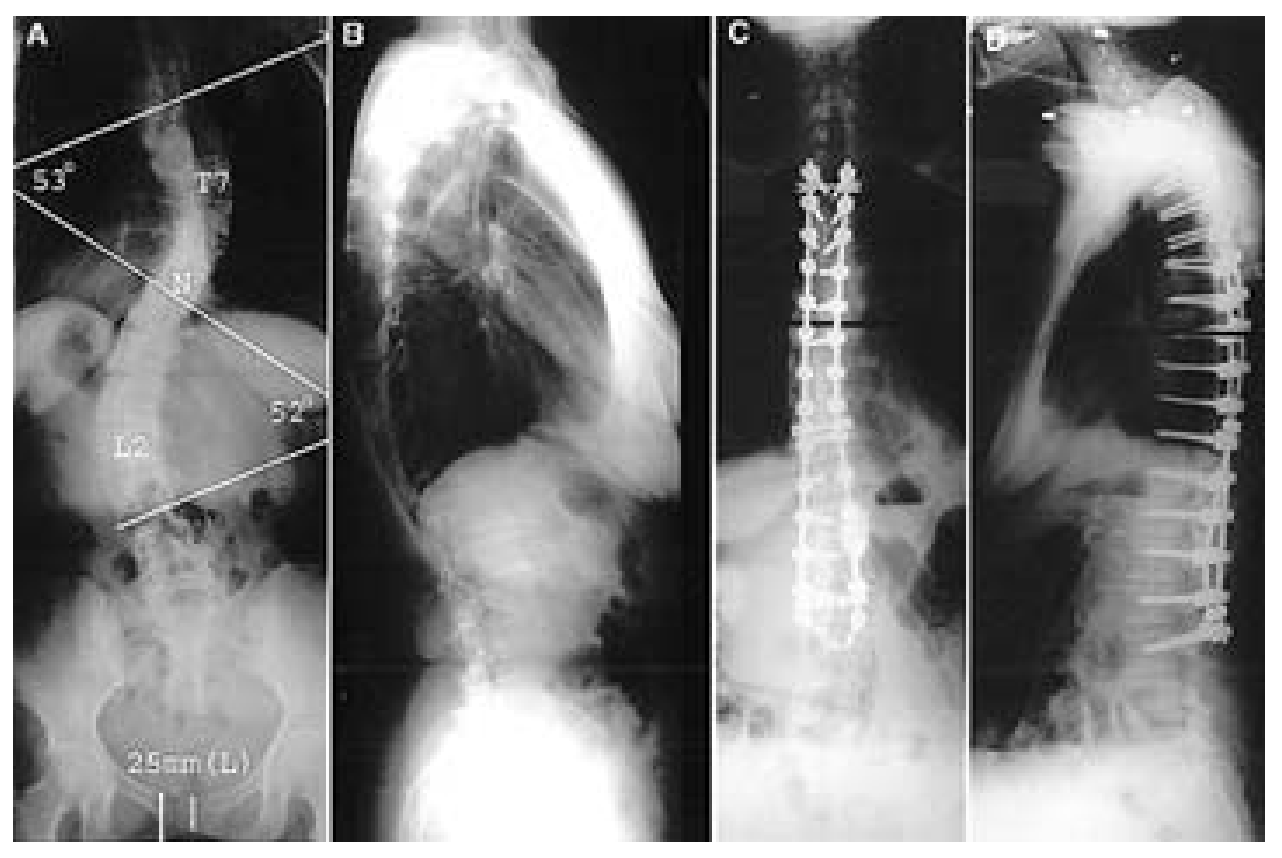

Fig. 1. Radiographic studies obtained in a 47-year-old woman who presented with adult degeneration of adolescent idiopathic scoliosis. A and B: Preoperative x-ray films revealing a thoracic curve of $53^{\circ}$ with the apex at T-7 and a lumbar curve of $52^{\circ}$ with the apex at L-2. The T-10 vertebra is the neutral vertebra $(\mathrm{N})$. C and D: Postoperative anteroposterior (AP) and lateral x-ray films demonstrating good correction of the scoliotic curvature. 


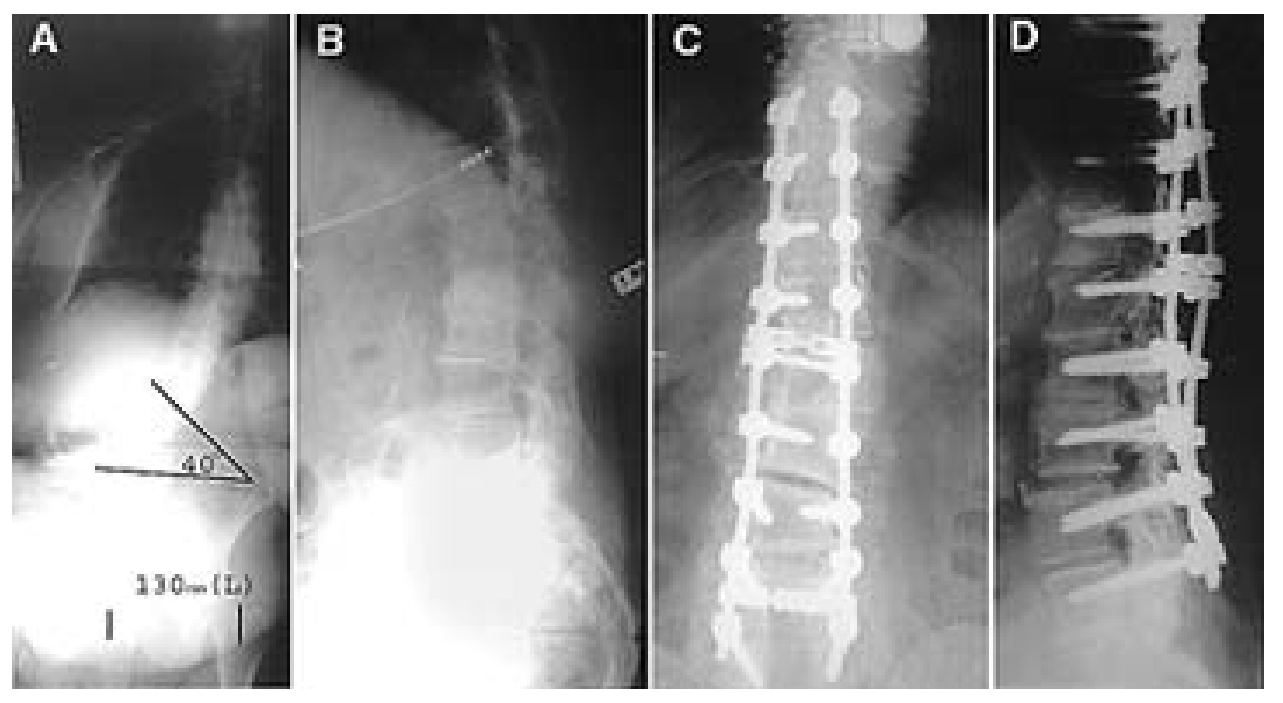

Fig. 2. Radiographic studies obtained in a 77-year-old woman who presented with intractable back pain and progressive scoliosis several years after multilevel lumbar laminectomy. A and B: Preoperative AP and lateral X-ray films. C and D: Postoperative AP and lateral x-ray films demonstrating good correction of the scoliosis and good placement of instrumentation.

Cerebrospinal fluid leaks occurred in two patients (both reoperative cases). Both presented with postural headaches, one several weeks and one 3 months after surgery. The first patient required readmission for placement of a lumbar drain, which led to resolution of the headaches. The other patient underwent dural repair, with subsequent headache resolution.

One patient suffered a pulmonary embolus complicated by congestive heart failure, which resolved after medical management. In one patient wound dehiscence developed 3 weeks after initial surgery. All cultures were negative, and after surgical repair of the wound, the patient did well.

\section{DISCUSSION}

Adult scoliosis is a challenging spinal disorder for the clinician. Compared with adolescent scoliosis, there are considerably fewer reports in the literature. Scoliosis in the adult patient is a different clinical entity than that in an adolescent. As in this study, the curves have apices that are predominantly in the lumbar spine, in contrast to the more common thoracic apices seen in adolescent scoliosis. Adult patients with scoliosis have bones that tend to be qualitatively and quantitatively weaker or osteoporotic, making instrumentation and fusion more difficult. ${ }^{1,6,30}$ Typical degenerative disc changes, spinal stenosis, and facet arthropathy are present, which can be exacerbated and in turn exacerbate scoliosis, leading to more rigid spines; these affected spines become more difficult to treat as the disease progresses and the patient ages. ${ }^{5,6,16,25,27,29,}$ 30,32,36 Associated sagittal-plane anomalies, including retrolistheses and spondylolistheses, can further lead to instability. These severe spinal deformities can compromise cardiovascular or pulmonary function, $5,6,14,25$, 27,29-32 increasing surgery-related morbidity and mortality. $5,6,29,30,32$ Many of the patients may be elderly or frail due to associated medical conditions. . $^{1,6,12,29-31}$ Compared with patients with adolescent idiopathic scoliosis, patients with adult scoliosis often complain of back pain.,12-14,16,20,21, $24,31,32$ As a result of these significant differences between adolescent and adult scoliosis, the therapeutic goals become different. In adolescents, the curves typically do not cause pain, and surgery is indicated to prevent a curve from progressing to a point at which it induces pain, causes significant cardiopulmonary compromise, and for the purposes of cosmesis. Ultimately, because these curves are more flexible than those in the adult disease, the goal is often to restore normal sagittal and coronal alignment. Adults often present with progressive back pain that interferes with activities of daily living, and the goals of surgery are to resolve the pain or to make it more controllable with medications, as well as to reduce their drug load. Because many of these patients present after having undergone multiple operations and failed fusions, one goal is to fuse the spine in as normal an anatomical position as possible. Often, a completely normal anatomical restoration is not a realistic therapeutic option. Cosmesis is not a common indication for the treatment of adult deformity.

Many authors have reported that the rigidity of these deformities necessitate anterior release to restore flexibility before instrumentation-augmented correction posteriorly can be successfully performed. ${ }^{5,7,16,36}$ Thoracotomies and laparotomies, however, are associated with significant morbidity and when combined with a posterior approach for complete deformity correction, affect a patient's ability to recuperate rapidly. To avoid these risks, we have performed anterior and posterior release procedures, reconstruction of the anterior weight-bearing columns, and instrumentation-assisted fusion via a posterior-only approach. Complications associated with the transthoracic approach include pulmonary complications (including atelectasis, pneumonia, pleural effusion, and pneumothorax), ${ }^{35,38}$ chylothorax, ${ }^{4}$ and postthoracotomy pain syn- 
drome. ${ }^{26}$ Risks associated with the retroperitoneal approach include injury to the abdominal viscera, the great vessels with associated risks of increased blood loss and blood transfusions, and the superior hypogastric nerve plexus leading to impotence and bladder dysfunction. ${ }^{11,38}$ Other complications include deep vein thrombosis due to retraction of venous structures, abdominal incisional hernia, and a prolonged postoperative ileus. ${ }^{38}$ Because our patients only undergo a single procedure, rehabilitation is faster and risks inherent in delayed mobilization are avoided.

The results in this series also highlight the utility of the PLIF in the operative treatment of adult scoliosis. In the surgical treatment of scoliosis the following procedures have been emphasized: instrumentation and fusion only of the posterior elements; ;, $, 6,8,13,17,21,27,29,30,32,36$ instrumentation and fusion only of the anterior elements via an anterior approach $; 5,6,13,17,19,20,27,29$ or combined procedures..$^{5-7,13,16,17 \text {, }}$ 19,29,30,36 To our knowledge, this is the only series involving an anterior release and fusion of all columns via a posterior-only approach in the setting of adult scoliosis. To accomplish this successfully, we use extended Gill laminectomies (removal of all posterior elements except for the pedicles and the associated superior articulating facet, thus disrupting the posterior elements completely). This allows us enough space in which to release the anterior column and then reconstruct the anterior and middle columns. This near-complete disruption of both anterior and posterior elements allows the spine to become flexible enough to be manipulated adequately from behind. In addition, the fusion rates after interbody fusion in conjunction with posterolateral fusion are significantly higher than those associated with posterolateral arthrodesis alone. ${ }^{9,28}$ Thus, this approach has several advantages: 1 ) the anterior weight-bearing columns can be adequately released, allowing for better spinal manipulation, and easier restoration of normal spinal alignment, and 2) we believe there to be a lower associated incidence of flat-back syndrome. By not performing an anterior procedure, we avoid the inherent risks associated with anterior staged procedures. Last, by adding interbody fusion to the placement of posterolateral instrumentation, a higher fusion rate can be achieved than when using posterolateral instrumentation alone.

The disadvantages of a posterior-only approach include less access to the anterior vertebral bodies and hindered ability to complete an anterior release successfully, even with the extensive posterior dissection we perform, which may lead to a less satisfying spinal alignment than would be possible when using combined procedures. Furthermore, to successfully complete a PLIF, the surgeon must work around the exiting spinal nerves, which can be particularly hazardous in the setting of a reoperation in which significant scar and bone graft have altered the anatomy.

Adjacent-segment failure has been reported in the lumbar spine after fusion for degenerative instability. ${ }^{2,10,13}$ In a prior study, we reported that the risk of such failure is increased particularly in patients who are older $(>60$ years of age), female, postmenopausal, osteoporotic, and with preoperative instability, long fusion segments, fusion masses not anchored to the thoracic or lumbar spine, and in whom coronal or sagittal balance has been lost. ${ }^{10}$ In this review, failure of the most cephalad adjacent segment is a significant problem: two (10\%) of the 20 surviving patients required a second procedure for this reason. One of these patients presented with progressive paraparesis, whereas the other presented during routine follow up. These cases illustrate the risks of adjacent-segment failure, as well as the need for consistent radiographic follow-up examination, because the second patient was asymptomatic but could have clearly been injured had the cephalad failure not been observed until later. Adjacent-segment failure in these patients was associated with severe osteoporosis and failure to extend the instrumentation above the apical segment of the thoracic kyphosis. This problem did not occur when the instrumentation was extended to the upper thoracic spine; we recommend considering the extension of the posterior instrumentation to the upper thoracic spine when treating patients at high risk for adjacent segment failure, especially in cases of exaggerated thoracic kyphosis commonly seen in older women.

\section{CONCLUSIONS}

We have found that in many cases of adult-onset degenerative or postsurgical scoliosis, a satisfactory multiplanar correction may be achieved using a single posterior approach combined with extended PLIF techniques. Cephalad adjacent-segment failure remains a significant problem in osteoporotic patients. To reduce that risk, we recommend the routine extension of posterior instrumentation to the upper thoracic spine in the subset of elderly osteoporotic patients.

\section{References}

1. Albert TJ, Purtill J, Mesa J, et al: Health outcome assessment before and after adult deformity surgery. A prospective study. Spine 20:2002-2005, 1995

2. Aota Y, Kumano K, Hirabayashi S: Postfusion instability at the adjacent segments after rigid pedicle screw fixation for degenerative lumbar spinal disorders. J Spinal Disord 8:464-473, 1995

3. Bernhardt M: Normal spinal anatomy: normal sagittal plane alignment, in Bridwell KH, DeWald RL (eds): The textbook of spinal surgery, ed 2. Philadelphia: Lippincott-Raven, 1997, pp 185-191

4. Bolesta MJ, Bohlman HH: Late sequelae of thoracolumbar fractures and fracture-dislocations: surgical treatment, in Frymoyer JW (ed): The adult spine: principles and practice. New York: Raven Press, 1991, Vol 2, pp 1331-1352

5. Bradford DS: Adult Scoliosis. Current concepts of treatment. Clin Orthop 229:70-87, 1988

6. Bradford DS, Tay BK, Hu SS: Adult scoliosis: surgical indications, operative management, complications, and outcomes. Spine 24:2617-2629, 1999

7. Byrd JA III, Scoles PV, Winter RB, et al: Adult idiopathic scoliosis treated by anterior and posterior spinal fusion. J Bone Joint Surg Am 69:843-850, 1987

8. Edgar MA: Back pain assessment from a long-term follow-up of operated and unoperated patients with adolescent idiopathic scoliosis. Spine 4:519-520, 1979

9. Enker P, Steffee AD: Interbody fusion and instrumentation. Clin Orthop 30:90-101, 1994

10. Etebar S, Cahill DW: Risk factors for adjacent-segment failure following lumbar fixation with rigid instrumentation for degenerative instability. J Neurosurg 90:163-169, 1999

11. Found EM, Weinstein JN: Surgical approaches to the lumbar 
spine, in Frymoyer JW (ed): The adult spine: principles and practice. New York: Raven Press, 1991, Vol 2, pp 1523-1534

12. Grubb SA, Lipscomb HJ, Coonrad RW: Degenerative adult onset scoliosis. Spine 13:241-245, 1988

13. Grubb SA, Lipscomb HJ, Suh PB: Results of surgical treatment of painful adult scoliosis. Spine 19:1619-1627, 1994

14. Jackson RP, Simmons EH, Stripinis D: Coronal and sagittal plane spinal deformities correlating with back pain and pulmonary function in adult idiopathic scoliosis. Spine 14: 1391-1397, 1989

15. Jackson RP, Simmons EH, Stripinis D: Incidence and severity of back pain in adult idiopathic scoliosis. Spine 8:749-756, 1983

16. Johnson JR, Holt RT: Combined use of anterior and posterior surgery for adult scoliosis. Orthop Clin North Am 19: 361-370, 1988

17. Kitahara $\mathrm{H}$, Inoue $\mathrm{S}$, Minami $\mathrm{S}$, et al: Long-term results of spinal instrumentation surgery for scoliosis five years or more after surgery, in patients over twenty-three years of age. Spine 14:744-749, 1989

18. Kostuik JP: Decision making in adult scoliosis. Spine 4: 521-525, 1979

19. Kostuik JP: Treatment of scoliosis in the adult thoracolumbar spine with special reference to fusion to the sacrum. Orthop Clin North Am 19:371-381, 1988

20. Kostuik JP, Carl A, Ferron S: Anterior Zielke instrumentation for spinal deformity in adults. J Bone Joint Surg Am 71: 898-912, 1989

21. Marchesi DG, Aebi M: Pedicle fixation devices in the treatment of adult lumbar scoliosis. Spine 17 (Suppl 8):304-309, 1992

22. Nachemson A: Adult scoliosis and back pain. Spine 4:513-517, 1979

23. Perennou D, Marcelli C, Herisson C, et al: Adult lumbar scoliosis. Epidemiologic aspects in a low-back pain population. Spine 19:123-128, 1994

24. Pritchett JW, Bortel DT: Degenerative symptomatic lumbar scoliosis. Spine 18:700-703, 1993

25. Simmons ED II, Kowalski JM, Simmons EH: The results of surgical treatment for adult scoliosis. Spine 18:718-724, 1993

26. Skubic JW, Kostuik JP: Thoracic pain syndromes and thoracic disc herniation, in Frymoyer JW (ed): The adult spine: principles and practice. New York: Raven Press, 1991, Vol 2, pp 1443-1461
27. Sponseller PD, Cohen MS, Nachemson AL, et al: Results of surgical treatment of adults with idiopathic scoliosis. J Bone Joint Surg Am 69:667-675, 1987

28. Suk SI, Lee CK, Kim WJ, et al: Adding posterior lumbar interbody fusion to pedicle screw fixation and posterolateral fusion after decompression in spondylolytic spondylolisthesis. Spine 22:210-220, 1997

29. Swank S, Lonstein JE, Moe JH, et al: Surgical treatment of adult scoliosis. A review of 222 cases. J Bone Joint Surg Am 63: 268-287, 1981

30. Takahashi S, Delecrin J, Passuti N: Surgical treatment of idiopathic scoliosis in adults: an age-related analysis of outcome. Spine 27:1742-1748, 2002

31. van Dam BE: Nonoperative treatment of adult scoliosis. Orthop Clin North Am 19:347-351, 1988

32. van Dam BE: Operative treatment of adult scoliosis with posterior fusion and instrumentation. Orthop Clin North Am 19: 353-359, 1988

33. Velis KP, Healey JH, Schneider R: Osteoporosis in unstable adult scoliosis. Clin Orthop 237:132-141, 1988

34. Weinstein SL, Ponseti IV: Curve progression in idiopathic scoliosis. J Bone Joint Surg Am 65:447-455, 1983

35. Westfall SH, Akbarnia BA, Merenda JT, et al: Exposure of the anterior spine. Technique, complications, and results in 85 patients. Am J Surg 154:700-704, 1987

36. Winter RB, Lonstein JE: Adult idiopathic scoliosis treated with Luque or Harrington rods and sublaminar wiring. J Bone Joint Surg Am 71:1308-1313, 1989

37. Winter RB, Lonstein JE, Denis F: Pain patterns in adult scoliosis. Orthop Clin North Am 19:339-345, 1988

38. Zileli M, Naderi S, Benzel EC, et al: Preoperative and surgical planning for avoiding complications, in Benzel EC (ed): Spine surgery: techniques, complication avoidance, and management. New York: Churchill Livingstone, 1999, pp 135-142

Manuscript received November 15, 2002.

Accepted in final form December 11, 2002.

Address reprint requests to: Caleb R. Lippman, M.D., Harbourside Medical Tower, 4 Columbia Drive, Suite 730, Tampa, Florida 33606-2351. email: caleb.lippman@mssm.edu. 